\title{
Artemisinin improves neurocognitive deficits associated with sepsis by activating the AMPK axis in the microglia.
}

\section{Shao-Peng Lin ( $\square$ linsp@qq.com )}

Second Affiliated Hospital of Guangzhou Medical College https://orcid.org/0000-0002-8488-8398

Jue-Xian Wei

the second affiliated hospital of Guangzhou medical university

\section{Shan Ye}

the second affiliated hospital of Guangzhou medical university

Jiasong $\mathrm{Hu}$

the second affiliated hospital of Guangzhou medical university

Jingyi Bu

the second affiliated hospital of Guangzhou medical university

\section{Lidong Zhu}

the second affiliated hospital of Guangzhou medical university

Qi Li

the second affiliated hospital of Guangzhou medical university

Haojun Liao

the second affiliated hospital of Guangzhou medical university

Yi Wu

the second affiliated hospital of Guangzhou medical university

Pei-Yi Lin

the second affiliated hospital of Guangzhou medical university

Sheng-Qiang Chen

East Region Military Command General Hospital

Xiao-Hui Chen

the second affiliated hospital of Guangzhou medical university

\section{Research}

Keywords: artemisinin, inflammation, cognitive dysfunction, microglia, AMP-Activated Protein Kinases

Posted Date: June 1st, 2020

DOI: https://doi.org/10.21203/rs.2.17969/v2 
License: (c) (i) This work is licensed under a Creative Commons Attribution 4.0 International License. Read Full License

Version of Record: A version of this preprint was published at Acta Pharmacologica Sinica on March 23rd, 2021. See the published version at https://doi.org/10.1038/s41401-021-00634-3. 


\section{Abstract}

Background and purpose: Artemisinin has been in use as an anti-malarial drug for almost half a century in the world. There is growing evidence that artemisinin also possesses potent anti-inflammatory and immunoregulatory properties. However, the efficacy of artemisinin treatment in neurocognitive deficits associated with sepsis remains unknown. Here, we evaluate the possible protective effects and explore the underlying mechanism of artemisinin on cognitive impairment resulting from sepsis.

Methods: Male C57BL/ 6 mice were pretreated with either vehicle or artemisinin, and then injected with LPS to establish an animal model of sepsis. The cognitive function was then assessed using the Morris water maze. Neuronal damage and neuroinflammation in the hippocampus were evaluated by immunohistochemical and ELISA analysis. Additionally, the protective mechanism of artemisinin was determined in vitro.

Results: The results showed that artemisinin preconditioning attenuated LPS-induced cognitive impairment, neural damage, and microglial activation in the mouse brain. The in vitro experiment revealed that artemisinin could reduce the production of pro-inflammatory cytokines and suppress the microglial migration in the BV2 microglia cells. Meanwhile, western blot demonstrated that artemisinin suppressed nuclear translocation of nuclear factor kappa-B and the expression of pro-inflammatory cytokines (i.e. tumor necrosis factor alpha, interleukin-6) by activating adenosine monophosphateactivated protein kinasea1 (AMPKa1) pathway. Furthermore, knock-down of AMPKa1 markedly abolished the anti-inflammatory effects of artemisinin.

Conclusion: Artemisinin is a potential therapeutic agent for sepsis-associated neuroinflammation and cognitive impairment, and its effect was probably mediated by the activation of AMPKa1 signalling pathway in microglia.

\section{Introduction}

Cognitive impairments are common complication derive from neuroinflammation in sepsis[1, 2]. Releases of inflammatory factors and metabolic alterations contribute to neuronal dysfunction and death during sepsis. Research suggests that neurocognitive dysfunction persists even after the patient has recovered from sepsis [3]. To date, the pathogenesis of cognitive impairment associated with sepsis has not been fully elucidated, and there is no effective preventive and therapeutic measures [5].

Since the discovery of artemisinin in the 1970s, artemisinin and its derivatives (artemisinins) have been used as first-line antimalarial drugs and saved millions of malarial patients worldwide[6, 7]. In recent years, considerable efforts have been made to explore the unique chemical and pharmacological properties of this remarkable phytochemical. Growing evidence reveals that artemisinin also possesses potent anti-inflammatory, anti-tumour, and anti-fibrosis properties[8-11]. We recently reported that artemisinin protects neuronal HT-22 cells from oxidative injury by activating the Akt pathway[12]. This suggests that artemisinin has a potential therapeutic effect on nervous system diseases. However, the 
role of artemisinin in neuroinflammation and cognitive dysfunction associated with sepsis remains unknown. In the present study, we evaluated the in vivo and in vitro therapeutic effects of artemisinin on neuroinflammation and cognitive dysfunction associated with sepsis. We further characterised the possible regulation and the protective mechanism of action of artemisinin.

\section{Materials And Methods}

\subsection{Experimental animals}

Male C57BL/6 mice (wt.15-20g, certificate no. SCXK2018-0002) were purchased from the Guangdong Laboratory Animal Central (Guangzhou, China) and housed under standard conditions. These mice were fed a standard laboratory diet. The animals were randomly divided into the following three groups: Sham group, LPS group and LPS +ART group. There were thirty mice in each group. Mice received the artemisinin (Sigma Aldrich, MO, USA) and LPS (Sigma Aldrich, MO, USA) intraperitoneal injection as shown in Fig. 1G. Artemisinin was dissolved in DMSO. The dose to be administered for artemisinin (30 mg/kg per day) and LPS (750 $\mathrm{gg} / \mathrm{kg}$ per day) was chosen based on previous studies[13-15]. The study protocol was approved by the Ethics Committee of the Second Affiliated Hospital of Guangzhou Medical University (Permit No. 2016-102) and all procedures were carried out according to the Guide for the Care and Use of Laboratory Animals of the National Institutes of Health, USA[16].

\subsection{Morris water maze}

To evaluate cognitive disorder and the therapeutic effects of artemisinin in mice, the Morris water maze (MWM) test was conducted as described previously $[15,17]$. First, the mice were trained to find a hidden platform in the maze within $60 \mathrm{~s}$. If the mice failed to find the platform within $60 \mathrm{~s}$, it was guided to the platform and allowed to stay on it for 10 s. The positioning navigation experiment was conducted after five days of LPS injection and the escape latency was recorded. On the 6th day of LPS injection, the space exploration experiment was performed where in the platform was removed from the pool. All mice were monitored for 60 s to observe the average swimming speed, distance of swimming and the percentage of total time in the targeted quadrant. Data were analysed using SMART 3.0 software designed for the MWM test (Harvard Apparatus Technology Co, Ltd., USA).

\subsection{Tissue preparation and histological analysis}

After the MWM test, mice were euthanised and transcardially perfused with phosphate-buffered saline (PBS), followed by a solution containing $4 \%$ paraformaldehyde. Sections of hippocampal tissue from the brain were prepared and fixed in $4 \%$ paraformaldehyde solution overnight. After being dehydrated in ethanol, the tissues were embedded in paraffin and cut into $4 \mu \mathrm{m}$ sections. Paraffin-embedded sections of the brain tissue were deparaffinised in xylene and rehydrated through descending grades of ethanol (Tianjin Sheng Winton Chemical Co., Ltd., Tianjin, China). Sections were then stained with haematoxylin and eosin for the visualization of tissue structures. The sections were examined under a light microscope 
(Nikon Technology Co, Ltd., Japan). The number of hippocampal Cornu Ammonis 1 (CA1) pyramidal neurons per $\mathrm{mm}^{2}$ was used to calculate the neuronal density.

\subsection{Immunofluorescence histochemistry}

After deparaffinization and rehydration, the slide was immersed in the EDTA antigen retrieval buffer and heated for antigen retrieval. The sections were blocked with $3 \%$ BSA for $30 \mathrm{~min}$ at room temperature, and then incubated with the primary antibody for cleaved caspase 3 (Wuhan Servicebio Biotechnology, Co., Ltd., China, GB11532, 1:500), ionised calcium binding adaptor molecule-1 (Iba-1, Abcam, Cambridge, UK. ab5076, 1:200), P2Y12 (Biowamp Life Science Lab, Co., Ltd., China, PAB32958, 1:100) and CD68 (Wuhan Servicebio Biotechnology, Co., Ltd., China, GB11067, 1:200), followed by fluorescent secondary antibodies. They were also incubated with DAPI solution at room temperature for $10 \mathrm{~min}$ to visualise the nucleus. The sections were imaged with a fluorescent microscope (Axio Observer Z1; Carl Zeiss AG, Germany).

A microglia morphology automated analysis was performed using the Fiji image J@ software as described previously[18, 19]. Measurements of microglia morphology was performed blinded to sample identity. Briefly, the microglial cells were randomly selected using the region of interest (ROI). The extra signal was eliminated using the brush tool and the binary images of microglial cells were obtained. In Fractal analysis, the outlined cell from the binary image was scan to obtain the hull and circle results including lacunarity, perimeter and radius data. In skeleton analysis, the binary image was converted into a skeletonized format. The plugin skeleton-analysis was performed, and the number of branches, endpoint and junctions were obtained.

\subsection{Cell culture}

BV2 microglial cells were cultured in DMEM (Thermo Fisher Scientific, MA, USA) supplemented with 10\% FBS (Thermo Fisher Scientific, MA, USA), 0.5\% penicillin, and 0.5\% streptomycin (Thermo Fisher Scientific, MA, USA) at $37^{\circ} \mathrm{C}$ with a humidified atmosphere of $5 \% \mathrm{CO} 2$ and $95 \%$ air. BV2 cells were then divided into three groups: control group, LPS group (treated with $100 \mathrm{ng} / \mathrm{mL}$ of LPS for 12 hours), and LPS+ART group (pretreated with $40 \mathrm{mM}$ of artemisinin for 2 hours and then co-treated with $100 \mathrm{ng} / \mathrm{mL}$ of LPS for another 12 hours).

\subsection{ELISA}

The expression levels of IL-6, TNF-a, IL-1a, II-1 $\beta$, MCP-1 and MIP-2 in the cell supernatants or hippocampus homogenates were measured using ELISA kits (Dakewe Bio-engineering Co., Ltd, Shenzhen, China) according to the manufacturer's instructions. The optical density values were measured at $450 \mathrm{~nm}$ by the microplate reader within $5 \mathrm{~min}$, and standard curves were plotted[20]. Both standards and samples were measured in triplicate. Different intervention times of artemisinin were conducted to find out the optimal intervention time (Additional file 1: Figure S1). 


\section{- Quantitative RT-PCR}

The mRNA expression for IL-6 and TNF-a was determined by qRT-PCR as described previously[21]. Briefly, total RNA was isolated using the TRIzoI ${ }^{\circledR}$ reagent (Invitrogen, USA) according to the manufacturer's protocol. Total RNA was reverse transcribed to synthesise cDNA using SuperScript III Reverse Transcription Kit (Invitrogen, USA). qRT-PCR was conducted using SYBR Green qPCR SuperMix (Invitrogen, USA) in the ViiA ${ }^{\text {TM }} 7$ Real-time PCR System (Applied Biosystems, USA). IL-6 was determined using 5'-CACATGTTCTCTGGGAAATCG-3' forward and 5'- TTGTATCTCTGGAAGTTTCAGATTGTT-3' reverse primers. TNF-a was determined using 5'-GCCACCACGCTCTTCTGTCTAC-3' forward and 5'GGGTCTGGGCCATAGAACTGAT-3' reverse primers. $\beta$-actin was determined using $5{ }^{\prime}$ GTACCACCATGTACCCAGGC-3' forward and 5'-AACGCAGCTCAGTAACAGTCC-3' reverse primers. Results were analysed using the $2^{-\triangle \Delta C t}$ method.

\section{- Western blot analysis}

Western blot analysis was performed on cell lysates according to standard protocols as described previously $[12,22]$. Briefly, the total protein of the cells was extracted with radio-immunoprecipitation assay (RIPA) lysis buffer (Kaiji Company, Shen Zhen, China) containing the complete protease inhibitor mixture and protein phosphatase inhibitor (Kaiji Company, Shen Zhen, China). Nuclear and cytoplasmic fractions of the cells were prepared using the Nuclear and Cytoplasmic Extraction kit (Mei5 Biotechnology, Co., Ltd. Beijing, China). Protein samples were separated by SDS-PAGE and transferred to polyvinylidene difluoride (PVDF) membranes. We then incubated the membrane with the diluted primary antibodies against AMPKa1 (Cell Signaling Technology Inc., Danvers, USA. cst 2795s, 1:1000), p-AMPKa1 (Cell Signaling Technology Inc., Danvers, USA. cst 4185s, 1:1000), inducible nitric oxide synthase (iNOS. Cell Signaling Technology Inc., Danvers, USA. cst 13120s, 1:1000), IL-6 (Cell Signaling Technology Inc., Danvers, USA. cst 12912, 1:1000), TNF-a (Abcam, Cambridge, UK. ab1793, 1:1000), $\beta$-actin (Cell Signaling Technology Inc., Danvers, USA. cst 4970s, 1:1000), NF-KB (Cell Signaling Technology Inc., Danvers, USA. cst 8242s, 1:1000), PCNA (Cell Signaling Technology Inc., Danvers, USA. cst 13100s, 1:1000) and aTubulin (Cell Signaling Technology Inc., Danvers, USA. cst 2125s, 1:1000) overnight at $4{ }^{\circ} \mathrm{C}$. The following day, diluted secondary antibodies were used to detect the corresponding primary antibodies. The relative expression level of proteins was analysed using Image-Pro Plus (IPP) 6.0 (Media Cybernetics Inc., Bethesda, MD, USA).

\subsection{Wound healing assay}

For the migration assay, BV2 microglial cells were seeded in 6-well plates and cultured until the formation of a $90 \%$ confluent monolayer. A linear wound was made by scratching the cell monolayer of BV2 cells with a sterile $200 \mu \mathrm{L}$ pipette tip. After washing with PBS to remove the cell fragments, the cells were incubated in a medium without FBS. The areas of the wound and cell migration were photographed by a phase-contrast microscope at $\mathrm{Oh}$ and $12 \mathrm{~h}$ after wounding. All images were analysed by IPP.

\subsection{Transwell}


A total of 100,000 BV2 cells were seeded in FBS-free DMEM media in the upper chamber of a transwell insert (Corning Incorporated, NY, USA). The lower chamber was filled with DMEM media supplemented with $10 \%$ FBS. After $12 \mathrm{~h}$ of incubation, the cells in the upper chamber were removed, and the cells that had invaded the membrane were fixed with $4 \%$ paraformaldehyde and stained with crystal violet for 20 min. Five randomly selected fields were captured with an optical microscope and the number of invading cells was counted.

\subsection{Small interfering RNA (siRNA) transfection}

AMPKa1 targeting siRNA for use in the BV2 cell lines was synthesised by Gene Pharma Company (Gene Pharma, China). The sequence of the specific AMPKa1 siRNA was 5'-GCAUAUGCUGCAGGUAGAUTT-3'. The sequence of scrambled control siRNA was 5'-UUCUCCGAACGUGUCACGUTT-3'. Cells were transfected with AMPKa1 siRNA (si-AMPKa1) or scrambled control siRNA (si-NC) 8h prior to further experimentation using Lipofectamine iMAX (Invitrogen, USA) according to the manufacturer's instructions.

\subsection{Immunocytochemistry staining}

Cells were fixed in BDCytofix/Cytoperm solution (BD Biosciences, NJ, USA) and permeabilised with $0.1 \%$ Triton-X. The cells were incubated overnight with primary antibody for nuclear factor kappa B (NF-KB). Cell Signaling Technology Inc., Danvers, USA. cst 8242s, 1:500) followed by labelling with FITC goat antirabbit IgG (Wuhan Servicebio Technology Co., Ltd, Wuhan, China. 1:400). Later the cells were incubated with $0.5 \mathrm{mg} / \mathrm{mL}$ DAPI for staining the nuclei. Images were obtained using a fluorescence microscope. To analyse the ratio of nuclear and cytoplasmic immune fluorescence of NF-KB, the percentage of cells showing high fluorescence in different areas of a cell (either nucleus or cytoplasm) was counted. A total of 200 cells were counted per group. Densitometry analysis of the nuclear translocation of NF-KB was expressed as a percentage of total cells.

\subsection{Statistical analysis}

The experimental results were expressed as mean \pm SD. Statistical analysis was performed with GraphPad Prism 7.0 software (San Diego, CA, USA) using one-way ANOVA followed by Tukey's post-hoc analysis. A value of $p$ less than 0.05 was considered as statistically significant.

\subsection{Materials}

BV2 microglial cells were purchased from Guangzhou Jennio Biotech Co., Ltd (Guangzhou, China). Artemisinin (purity $>98 \%$ ), LPS and DMSO were from Sigma Aldrich (MO, USA). Iba-1 (ab5076) and TNF-a (ab1793) antibodies were purchased from Abcam (Cambridge, UK). AMPKa1 (cst 2795s), p-AMPKa1 (cst 4185s), iNOS (cst 13120s), IL-6 (cst 12912), NF-KB (cst 8242s), PCNA (cst 13100s), a-Tubulin (cst 2125s) and $\beta$-actin (cst 4970s) antibodies were purchased from Cell Signaling Technology Inc (Danvers, USA). Cleaved caspase 3 (GB11532) and CD68 (GB11067) antibodies was purchased from Wuhan Servicebio Biotechnology, Co., Ltd. (Wuhan, China). P2Y12 antibody (PAB32958) was purchased from Biowamp Life 
Science Lab, Co., Ltd. (Wuhan, China). TNF-a, IL-6, IL-1a, IL-1 $\beta$, MCP-1 and MIP-2 ELISA kits were purchased from Dakewe Bio-engineering Co., Ltd (Shenzhen, China).

\section{Results}

\subsection{Artemisinin ameliorated neuronal cell death and improved cognitive impairment in LPS-induced murine neuroinflammatory model}

Following LPS-stimulated, LPS group mice exhibited a 90\% 7-days survival rate. In contrast, the survival rate among artemisinin treatment group mice was $100 \%$. Morris water maze training was applied to assess the efficacy of artemisinin treatment in LPS-induced cognitive impairment. Five days after systemic injection of LPS, a positioning navigation experiment was performed to observe the cognitive function of mice. As shown in Fig.1A and B, compared to sham group, mice exposed to LPS exhibited a significantly prolonged escape latency to find the platform, while those pretreated with artemisinin exhibited a shorter escape latency $(p<0.05)$. On the 6th day, the space exploration experiment was performed in the absence of the platform. Our data demonstrated that the percentage of total time in the targeted quadrant was significantly reduced in the LPS-challenged group compared with the sham group, while mice in the artemisinin-pretreated group spent significantly more time in the targeted quadrant than the LPS-challenged group $(p<0.05)$. There was no significant difference between the three groups of mice with regard to the average speed nor the distance travelled in the space exploration test $(p>0.05)$. These findings suggest that artemisinin could improve LPS-induced memory and cognitive impairment.

The hippocampus is closely related to cognitive and memory function. To determine whether artemisinin could affect neuronal survival in the hippocampal region and improve cognition, we performed haematoxylin and eosin staining of the hippocampus. It was found that LPS induced a significant reduction in the number of hippocampal CA1 neurons. Notably, artemisinin pretreatment could ameliorate the neuronal cell death in the CA1 region (Fig.1C, D). Cleaved caspase 3 staining also demonstrated that artemisinin pretreatment could protects CA1 neuron from LPS-induced injury (Fig.1E, F). Meanwhile, Iba-1 immunoreactive microglia was abundant in the hippocampus of the LPS group. Artemisinin administration induced a dramatic decrease in Iba-1 positive cells in the hippocampal region (Fig. 2A, B). Microglia morphology is associated with microglial activation[18]. The fractal analysis was used to evaluate the changes in microglia shape. The lacunarity, perimeter and radius were significant decreased in the LPS group compared to control group. Furthermore, the decreased in lacunarity, perimeter and radius were reversed in artemisinin pretreatment group. The skeleton analysis was used to evaluate the differences of ramified structure of the microglia cell by measuring the branches, junctions and endpoints. As predicted, LPS induced a significant decrease in the length of branches and the number of endpoint and junctions, which were reversed by artemisinin pretreatment (Fig. 2D, E). What is more, specific microglia markers (P2Y12) and activation markers (CD68) double-staining were analyzed in hippocampal region (Fig. 2F). Consistent with the microglia morphology analysis, artemisinin preconditioning attenuated LPS-induced activated microglia significantly (Fig. 2G). The inflammatory response in hippocampus was also evaluated through the expression of TNF-a. ELISA analysis found 
that the exposure to LPS resulted in a significant secretion of TNF- $\alpha$ and artemisinin could reverse this inflammatory response (Fig. 2C). This data demonstrated that artemisinin could ameliorate the hippocampal neuron cell death and improve cognitive impairment caused by LPS via suppressing microglial activation.

\subsection{Artemisinin regulated the pro-inflammatory cytokines and inhibited migratory ability of BV2 microglia}

Microglial cell activation and proliferation precedes the onset of CNS injury[23]. Activated microglia produce a wide range of pro-inflammatory mediators. To investigate the effects of artemisinin on LPSactivated BV2 microglia, ELISA assay was employed to determine the level of inflammatory cytokines in the supernatant of BV2 cells. We observed a marked release of pro-inflammation cytokines (TNF-a, IL-6, IL-1 $a$ and IL-1 $\beta$ ) following 12 hours of LPS stimulation, which was however abolished by artemisinin treatment (Fig.3). Furthermore, mRNA and protein expression changes of TNF-a and IL- 6 were confirmed with qRT-PCR and western blot, respectively. Our data showed significant increase in mRNA expression of IL- 6 and TNF-a in the LPS-activated group, which was markedly inhibited by artemisinin (Fig.4A, B). Similarly, western blot confirmed that artemisinin treatment significantly inhibited the protein expression of IL-6 and TNF- $a$ after LPS stimulation (Fig.4 C, D). To observe the effect of artemisinin on microglial cell motility, both wound-healing assay and transwell chamber were employed. Compared with normal control, LPS stimulation efficiently enhanced the migratory ability of BV2 cells. However, such an effect was diminished in the presence of artemisinin (Fig.5A-D). ELISA assay demonstrated that artemisinin could reversed the increase of the chemokines MCP-1 and MIP-2 induced by LPS stimulation (Fig.5E, F). The above findings indicate that artemisinin could reduce the production of pro-inflammatory cytokines and suppress the microglial migration.

\subsection{AMPKa1 pathway is involved in the anti-inflammatory effect of artemisinin}

We next investigated whether the AMPKa1 pathway was involved in the anti-inflammatory effect of artemisinin. Preliminary western blot demonstrated that artemisinin could activate AMPKa1 in a dosedependent manner (Fig.6A). We further discovered that activation of AMPKa1 by artemisinin significantly attenuates LPS-induced inflammatory pathways in BV2 cells. Firstly, immunofluorescence and western blot analysis revealed that LPS stimulated nuclear translocation of NF-KB, whereas pretreatment with artemisinin markedly decreased the nuclear translocation of NF-KB (Fig. $6 \mathrm{C}$ and D). Western blot analysis showed that the LPS-induced up-regulation of iNOS, IL-6, and TNF-a expression was also reversed by artemisinin pretreatment (Fig.6B). These results further suggest that activation of AMPKa1 by artemisinin significantly attenuated the activation of NF-KB inflammatory pathway induced by LPS.

The essential role of AMPKa1 activation in the anti-inflammatory effect of artemisinin was confirmed using si-RNA specific to AMPKa1. As shown in Fig.7A, immune blotting showed excellent knock-down efficiency of si-RNA specific to AMPKa1. We observed that artemisinin inhibited the LPS-induced increase in TNF-a protein production, but this effect was significantly reduced after transfection with AMPKa1 siRNA (Fig. 7B). ELISA analysis demonstrated that the anti-inflammatory effect of artemisinin was 
partially abolished by si-RNA specific to AMPKa1 (Fig. 7C and D). Therefore, the results suggest that artemisinin attenuated the LPS-induced inflammation by activation of AMPKa1 .

\section{Discussion}

In our study, artemisinin exhibited powerful anti-inflammatory and neuroprotective activities in the sepsis model. This protection was mediated by the activation of AMPKa1 signalling in the microglia (Fig.8).

Cognitive impairment is an essential manifestation of sepsis[4]. Unfortunately, the treatment outcomes remain unsatisfactory. Many patients continue to have cognitive dysfunction after the sepsis has healed[3]. The cognitive impairment of sepsis was caused by the immune response to bacterial LPS or other endotoxic components of bacteria without a direct central nervous system infection[24]. Therefore, we chose systemic LPS-injection to induce the experimental sepsis model and relevant experiments were conducted using this model. Our results have shown that artemisinin could improve the cognitive function in sepsis mice in the Morris water maze test. Artemisinin preconditioned mice exhibited better spatial learning-memory than the LPS-challenged group. It has been reported that artemisinin B, one of the compounds isolated from Artemisia annuaL., improves spatial memory in the water maze test in a mouse model of dementia [25]. This study provides preliminary evidence for the neuroprotective effect of artemisinins against neurodegenerative diseases. Our results showed that artemisinin could improve cognitive dysfunction in the sepsis mice model and further illustrate the effects of artemisinin on neurological disorders.

A previous clinical study of brain tissue has found an association between activation of microglia and sepsis[26]. Excessive activation of microglia has been demonstrated as one of the main mechanisms of pathogenesis in neurocognitive deficits associated with sepsis[5, 27]. Activation of microglia releases multiple inflammatory factors resulting in hippocampal neuronal damage which ultimately leads to cognitive dysfunction[25]. Therefore, suppression of microglial activation has therapeutic potential in the treatment of neurocognitive deficits associated with sepsis[28,29]. Pathological changes in the hippocampus were measured to detect neuronal survival and microglial activation in our study. Our results showed that artemisinin inhibited LPS-induced activation of microglia and neuronal death in the hippocampal CA1 region. Taken together, this study demonstrates that artemisinin could restore hippocampus-mediated cognition by inhibiting the microglial cell activation and neuronal death in the CA1 region.

To further evaluate the potential pharmacological mechanism of artemisinin in microglia, ELISA technology was applied to detect the expression levels of cytokines in the BV2 cell supernatants. We observed a marked release of pro-inflammatory cytokines (TNF-a, IL-6, IL-1a and IL-1 $\beta$ ) following LPS stimulation for 12 hours, which was abolished by artemisinin treatment. Previous in-vitro experiments have also shown that artemisinins can inhibit the TNF- $a$ and IL- 6 release from LPS stimulated BV2 cells[30], which is in accordance with our result. Neuroinflammation, mainly characterised by the activation of microglial cells and the massive production of pro-inflammatory cytokines is found to be 
involved in cognitive impairment associated with sepsis[31, 32]. Excessive neuroinflammation would cause neuronal damage and cognitive impairment by causing an over-production of pro-inflammatory cytokines[33-35]. Inhibition of over-activated microglia appears to be a potential therapeutic strategy in cognitive impairment associated with sepsis[27]. Based on our study, it can be concluded that artemisinins have appreciable anti-inflammatory effects and thus decrease the toxic effects of proinflammatory cytokines on neurons.

ELISA assay also revealed that artemisinin inhibited the chemokines (MCP-1 and MIP-2) which were induced by LPS stimulation. Chemokines are crucial factors for an early inflammatory response that evoke migration of the microglial cells[36]. Microglial cells migrate to the site of injury or lesion, and play a pivotal role in the occurrence, and development of inflammation[37]. In this study, immunofluorescence of Iba-1, wound healing assay and transwell migration assay were performed to test whether artemisinin could reduce the migratory capacity of microglia cells. The immunofluorescence test for lba-1 showed that artemisinin significantly inhibited LPS-induced migration of microglia in the brain. Wound healing assay and transwell migration assay also revealed that artemisinin inhibited the migratory ability of BV2 microglia. Our data suggests that the anti-inflammatory effect of artemisinin might be exerted by suppressing microglial cell motility via inhibition of chemokine activity.It was reported that AMPK was involved in the pathogenesis of cognitive impairment in sepsis and activated AMPK pathway could attenuation the cognitive impairment in sepsis mice[38, 39]. Recent studies also found that AMPK was an important therapeutic target of artemisinin in some diseases model, such as atherosclerosis and Alzheimer's mouse model[40, 41]. However, whether AMPK was a therapeutic target of artemisinin in neuroinflammation resulting from sepsis remains unknown. Therefore, we explored whether the effect of artemisinin in neuroinflammation resulting from sepsis are through the regulation of AMPK pathway. AMPK is a highly conserved serine/threonine protein kinase present in eukaryotic cells[42, 43]. AMPK participates in multiple biological processes by modulating cellular energy homeostasis. It has become an attractive drug target for diabetes, stroke, and tumour [44]. Recent studies found that AMPK is also involved in inflammatory response through regulation of NF-KB signalling pathway[45]. It has been reported that phosphorylated-AMPK inhibits the activation of NF-KB and decreases the production of IL-6, TNF- $\alpha$ and IL-1 $\beta[46-49]$. These results suggest that AMPK phosphorylation may also serve as an important therapeutic target for inflammation $[42,50]$. However, whether artemisinin inhibits neuroinflammation through the AMPK/NF-KB pathway remains unexplored. Firstly, our study demonstrated that artemisinin could activate the AMPK in a dose-dependent manner in microglia cells. Furthermore, pretreatment with artemisinin markedly decreased the nuclear translocation of NF-KB in BV2 cells. PCR, western Blot and ELISA analysis showed that LPS-induced up-regulation of IL- 6 and TNF-a mRNA, and both proteins were also reversed by artemisinin pretreatment. Therefore, the present study suggests that artemisinin increased the expression of AMPK and blocked the nuclear translocation of NFKB where it can initiate transcription by binding to gene promoter elements of IL- 6 and TNF- $a$. These results suggest that the AMPK/NF-KB pathway is involved in the anti-inflammatory activity of artemisinin in neuroinflammation. Previous research has shown that artemisinin could regulate AMPK pathway, hence itis an attractive drug target for cancer and oxidative stress injury[51, 52]. We demonstrate for the 
first time in the current study that AMPK pathway can be the therapeutic target of artemisinin for the treatment of neuroinflammation.

\section{Conclusion}

In summary, our data has proved for the first time that artemisinin inhibits hippocampal neuronal death and cognitive impairment by inhibiting microglial activation in the sepsis mouse model. In addition, it was found that artemisinin significantly increased the AMPKa1 to inhibit LPS-induced neuroinflammatory response in BV2 microglial cells. Our finding indicate that artemisinin may be a potential preventive and therapeutic agent in neurocognitive deficits associated with sepsis.

\section{Declarations}

\section{- Ethical Approval and Consent to participate}

The studies were approved by the Ethics Committee of the Second Affiliated Hospital of Guangzhou Medical University.

\section{- Consent for publication}

Not applicable

\section{- Availability of supporting data}

Additional file 1: Anti-inflammatory effect of artemisinin at different intervention time (Figure S1). Competing interests

All authors declare that there are no conflicts of interest.

\section{- Funding}

This work was partly supported by the National Natural Science Foundation of China (grant No.81641088), the Natural Science Foundation of Guangdong Province (grant No.2016A030310267 and 2017B030311019), the Science and Technology Planning Project of Guangdong Province (grant No.2015A030302091), the Science and Technology Planning Project of Guangzhou (grant No.201607010160), the Nature Science Foundation in Guangzhou Medical University (grant No.2015C14), the Key Medical Disciplines and Specialities Program of Guangzhou(2017-2019) .

\section{- Authors' contributions}

X.-H.C., P.-Y.L and S.P.L. designed the experiments. J.-X.W., J.H., J.B., L.Z., Q.L, H.L, Y.W, and S.-Q.C. conducted the experiments. S.-P.L. and S.Y. wrote the manuscript. All the authors analyzed the data, revised the manuscript, and approved the final manuscript.

\section{- Acknowledgements}


Guangzhou Institute of Cardiovascular Disease, the Second Affiliated Hospital of Guangzhou Medical University has provided us with some experimental instruments.

\section{Abbreviations}

LPS, Lipopolysaccharides; AMPK, AMP-Activated Protein Kinases; NF-kB, nuclear factor kappa-B; TNF-a, tumor necrosis factor alpha, IL-6, interleukin-6; MWM, Morris water maze; CA1, Cornu Ammonis 1; CNS, central nervous system.

\section{References}

1. Zhang LN, Wang XT, Ai YH, Guo QL, Huang L, Liu ZY, Yao B: Epidemiological features and risk factors of sepsis-associated encephalopathy in intensive care unit patients: 2008-2011.Chin Med J (Engl) 2012, 125:828-831.

2. Silva AYO, Amorim EA, Barbosa-Silva MC, Lima MN, Oliveira HA, Granja MG, Oliveira KS, Fagundes PM, Neris RLS, Campos RMP, et al: Mesenchymal Stromal Cells Protect the Blood-Brain Barrier, Reduce Astrogliosis, and Prevent Cognitive and Behavioral Alterations in Surviving Septic Mice.Crit Care Med 2020, 48:e290-e298.

3. Widmann CN, Heneka MT: Long-term cerebral consequences of sepsis.Lancet Neurol 2014, 13:630636.

4. Stubbs DJ, Yamamoto AK, Menon DK: Imaging in sepsis-associated encephalopathy-insights and opportunities.Nat Rev Neurol 2013, 9:551-561.

5. Sonneville R, de Montmollin E, Poujade J, Garrouste-Orgeas M, Souweine B, Darmon M, Mariotte E, Argaud L, Barbier F, Goldgran-Toledano D, et al: Potentially modifiable factors contributing to sepsisassociated encephalopathy.Intensive Care Med 2017, 43:1075-1084.

6. Tu Y: Artemisinin-A Gift from Traditional Chinese Medicine to the World (Nobel Lecture).Angew Chem Int Ed Eng/ 2016, 55:10210-10226.

7. Miller LH, Su X: Artemisinin: discovery from the Chinese herbal garden.Cel/ 2011, 146:855-858.

8. Das AK: Anticancer Effect of AntiMalarial Artemisinin Compounds.Ann Med Health Sci Res 2015, 5:93-102.

9. Chen W, Li FF, Li C, Sui JK, Meng QF, Li XL, Li H, Li CH, Li YB: Artemisinin ameliorates the symptoms of experimental autoimmune myasthenia gravis by regulating the balance of TH1 cells, TH17 cells and Treg cells.J Biol Regul Homeost Agents 2018, 32:1217-1223.

10. Tilaoui M, Mouse HA, Jaafari A, Zyad A: Differential effect of artemisinin against cancer cell lines.Nat Prod Bioprospect 2014, 4:189-196.

11. Liu X, Lu J, Liao Y, Liu S, Chen Y, He R, Men L, Lu C, Chen Z, Li S, et al: Dihydroartemisinin attenuates lipopolysaccharide-induced acute kidney injury by inhibiting inflammation and oxidative stress.Biomed Pharmacother 2019, 117:109070. 
12. Lin SP, Li W, Winters A, Liu R, Yang SH: Artemisinin Prevents Glutamate-Induced Neuronal Cell Death Via Akt Pathway Activation.Front Cell Neurosci 2018, 12:108.

13. Yao Y, Guo Q, Cao Y, Qiu Y, Tan R, Yu Z, Zhou Y, Lu N: Artemisinin derivatives inactivate cancerassociated fibroblasts through suppressing TGF-beta signaling in breast cancer.J Exp Clin Cancer Res 2018, 37:282.

14. Xie LH, Li Q, Zhang J, Weina PJ: Pharmacokinetics, tissue distribution and mass balance of radiolabeled dihydroartemisinin in male rats.Malar $J$ 2009, 8:112.

15. Liu W, Huang S, Li Y, Zhang K, Zheng X: Suppressive effect of glycyrrhizic acid against lipopolysaccharide-induced neuroinflammation and cognitive impairment in C57 mice via toll-like receptor 4 signaling pathway. Food Nutr Res 2019, 63.

16. National Research Council (U.S.). Committee for the Update of the Guide for the Care and Use of Laboratory Animals., Institute for Laboratory Animal Research (U.S.), National Academies Press (U.S.): Guide for the care and use of laboratory animals. 8th edn. Washington, D.C.: National Academies Press; 2011.

17. Zhao J, Bi W, Xiao S, Lan X, Cheng X, Zhang J, Lu D, Wei W, Wang Y, Li H, et al: Neuroinflammation induced by lipopolysaccharide causes cognitive impairment in mice.Sci Rep 2019, 9:5790.

18. Vargas-Caraveo A, Sayd A, Robledo-Montana J, Caso JR, Madrigal JLM, Garcia-Bueno B, Leza JC: Toll-like receptor 4 agonist and antagonist lipopolysaccharides modify innate immune response in rat brain circumventricular organs.J Neuroinflammation 2020, 17:6.

19. Young K, Morrison H: Quantifying Microglia Morphology from Photomicrographs of Immunohistochemistry Prepared Tissue Using ImageJ.J Vis Exp 2018.

20. Lin SP, Ye S, Chen XH, Jiang HL, Mao HF, Chen MT, Ma QJ, Long Y, Fan Y, Lin PY: Increased expression of microRNA-21 in peripheral blood mediates the down-regulation of IFN-gamma and increases the prevalence of stroke-associated infection.J Neurol Sci 2016, 366:235-239.

21. Lin SP, Ye S, Long Y, Fan Y, Mao HF, Chen MT, Ma QJ: Circular RNA expression alterations are involved in OGD/R-induced neuron injury.Biochem Biophys Res Commun 2016, 471:52-56.

22. Peng X, Wang Y, Li H, Fan J, Shen J, Yu X, Zhou Y, Mao H: ATG5-mediated autophagy suppresses NFkappaB signaling to limit epithelial inflammatory response to kidney injury. Cell Death Dis 2019, 10:253.

23. Ponomarev ED, Shriver LP, Maresz K, Dittel BN: Microglial cell activation and proliferation precedes the onset of CNS autoimmunity.J Neurosci Res 2005, 81:374-389.

24. Schedlowski M, Engler H, Grigoleit JS: Endotoxin-induced experimental systemic inflammation in humans: a model to disentangle immune-to-brain communication.Brain Behav Immun 2014, 35:1-8.

25. Qiang W, Cai W, Yang Q, Yang L, Dai Y, Zhao Z, Yin J, Li Y, Li Q, Wang Y, et al: Artemisinin B Improves Learning and Memory Impairment in AD Dementia Mice by Suppressing Neuroinflammation.Neuroscience 2018, 395:1-12.

26. Lemstra AW, Groen in't Woud JC, Hoozemans JJ, van Haastert ES, Rozemuller AJ, Eikelenboom P, van Gool WA: Microglia activation in sepsis: a case-control study.J Neuroinflammation 2007, 4:4. 
27. Pan C, Si Y, Meng Q, Jing L, Chen L, Zhang Y, Bao H: Suppression of the RAC1/MLK3/p38 Signaling Pathway by beta-Elemene Alleviates Sepsis-Associated Encephalopathy in Mice.Front Neurosci 2019, 13:358.

28. Beggs S, Salter MW: SnapShot: Microglia in Disease.Cell 2016, 165:1294-1294 e1291.

29. Sui DM, Xie Q, Yi WJ, Gupta S, Yu XY, Li JB, Wang J, Wang JF, Deng XM: Resveratrol Protects against Sepsis-Associated Encephalopathy and Inhibits the NLRP3/IL-1beta Axis in Microglia.Mediators Inflamm 2016, 2016:1045657.

30. Zhu C, Xiong Z, Chen X, Peng F, Hu X, Chen Y, Wang Q: Artemisinin attenuates lipopolysaccharidestimulated proinflammatory responses by inhibiting NF-kappaB pathway in microglia cells. PLoS One 2012, 7:e35125.

31. Block ML, Calderon-Garciduenas L: Air pollution: mechanisms of neuroinflammation and CNS disease. Trends Neurosci 2009, 32:506-516.

32. Heneka MT, Carson MJ, El Khoury J, Landreth GE, Brosseron F, Feinstein DL, Jacobs AH, Wyss-Coray T, Vitorica J, Ransohoff RM, et al: Neuroinflammation in Alzheimer's disease.Lancet Neuro/ 2015, 14:388-405.

33. Xanthos DN, Sandkuhler J: Neurogenic neuroinflammation: inflammatory CNS reactions in response to neuronal activity.Nat Rev Neurosci 2014, 15:43-53.

34. Tang Y, Le W: Differential Roles of M1 and M2 Microglia in Neurodegenerative Diseases.Mol Neurobio/ 2016, 53:1181-1194.

35. Wang YW, Zhou Q, Zhang X, Qian QQ, Xu JW, Ni PF, Qian YN: Mild endoplasmic reticulum stress ameliorates lipopolysaccharide-induced neuroinflammation and cognitive impairment via regulation of microglial polarization.J Neuroinflammation 2017, 14:233.

36. Piao HZ, Choi IY, Park JS, Kim HS, Cheong JH, Son KH, Jeon SJ, Ko KH, Kim WK: Wogonin inhibits microglial cell migration via suppression of nuclear factor-kappa B activity.Int Immunopharmacol 2008, 8:1658-1662.

37. Fang Y, Wang J, Yao L, Li C, Wang J, Liu Y, Tao X, Sun H, Liao H: The adhesion and migration of microglia to beta-amyloid (Abeta) is decreased with aging and inhibited by Nogo/NgR pathway.J Neuroinflammation 2018, 15:210.

38. Luo L, Wu J, Qiao L, Lu G, Li J, Li D: Sestrin 2 attenuates sepsis-associated encephalopathy through the promotion of autophagy in hippocampal neurons.J Cell Mol Med 2020.

39. Zhuang X, Yu Y, Jiang Y, Zhao S, Wang Y, Su L, Xie K, Yu Y, Lu Y, Lv G: Molecular hydrogen attenuates sepsis-induced neuroinflammation through regulation of microglia polarization through an mTORautophagy-dependent pathway.Int Immunopharmaco/2020, 81:106287.

40. Cao Q, Du H, Fu X, Duan N, Liu C, Li X: Artemisinin Attenuated Atherosclerosis in High-Fat Diet-Fed ApoE-/- Mice by Promoting Macrophage Autophagy Through the AMPK/mTOR/ULK1 Pathway.J Cardiovasc Pharmacol 2020, 75:321-332.

41. Li S, Zhao X, Lazarovici P, Zheng W: Artemether Activation of AMPK/GSK3beta(ser9)/Nrf2 Signaling Confers Neuroprotection towards beta-Amyloid-Induced Neurotoxicity in 3xTg Alzheimer's Mouse 
Model.Oxid Med Cell Longev 2019, 2019:1862437.

42. Lin SC, Hardie DG: AMPK: Sensing Glucose as well as Cellular Energy Status. Cell Metab 2018, 27:299-313.

43. Garcia D, Shaw RJ: AMPK: Mechanisms of Cellular Energy Sensing and Restoration of Metabolic Balance.Mol Cell 2017, 66:789-800.

44. Jiang T, Yu JT, Zhu XC, Wang HF, Tan MS, Cao L, Zhang QQ, Gao L, Shi JQ, Zhang YD, Tan L: Acute metformin preconditioning confers neuroprotection against focal cerebral ischaemia by preactivation of AMPK-dependent autophagy.Br J Pharmaco/2014, 171:3146-3157.

45. Jian M, Kwan JS, Bunting M, Ng RC, Chan KH: Adiponectin suppresses amyloid-beta oligomer (Abeta0)-induced inflammatory response of microglia via AdipoR1-AMPK-NF-kappaB signaling pathway.J Neuroinflammation 2019, 16:110.

46. Cacicedo JM, Yagihashi N, Keaney JF, Jr., Ruderman NB, Ido Y: AMPK inhibits fatty acid-induced increases in NF-kappaB transactivation in cultured human umbilical vein endothelial cells.Biochem Biophys Res Commun 2004, 324:1204-1209.

47. Thirupathi A, de Souza CT: Multi-regulatory network of ROS: the interconnection of ROS, PGC-1 alpha, and AMPK-SIRT1 during exercise.J Physiol Biochem 2017, 73:487-494.

48. Afonina IS, Zhong Z, Karin M, Beyaert R: Limiting inflammation-the negative regulation of NF-kappaB and the NLRP3 inflammasome.Nat Immuno/ 2017, 18:861-869.

49. Salt IP, Palmer TM: Exploiting the anti-inflammatory effects of AMP-activated protein kinase activation.Expert Opin Investig Drugs 2012, 21:1155-1167.

50. O'Neill LA, Hardie DG: Metabolism of inflammation limited by AMPK and pseudo-starvation.Nature 2013, 493:346-355.

51. Cheng C, Wang T, Song Z, Peng L, Gao M, Hermine O, Rousseaux S, Khochbin S, Mi JQ, Wang J: Induction of autophagy and autophagy-dependent apoptosis in diffuse large B-cell lymphoma by a new antimalarial artemisinin derivative, SM1044. Cancer Med 2018, 7:380-396.

52. Zhao X, Fang J, Li S, Gaur U, Xing X, Wang H, Zheng W: Artemisinin Attenuated Hydrogen Peroxide (H2O2)-Induced Oxidative Injury in SH-SY5Y and Hippocampal Neurons via the Activation of AMPK Pathway.Int J Mol Sci 2019, 20.

\section{Figures}


(A)
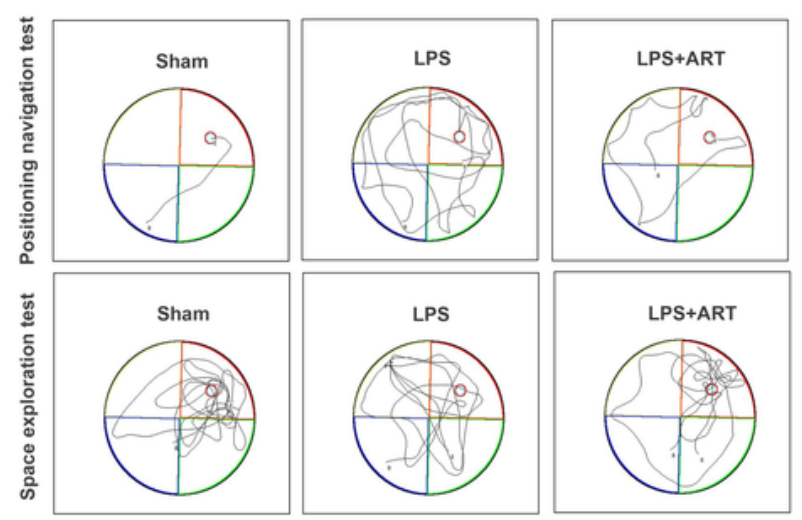

(C)

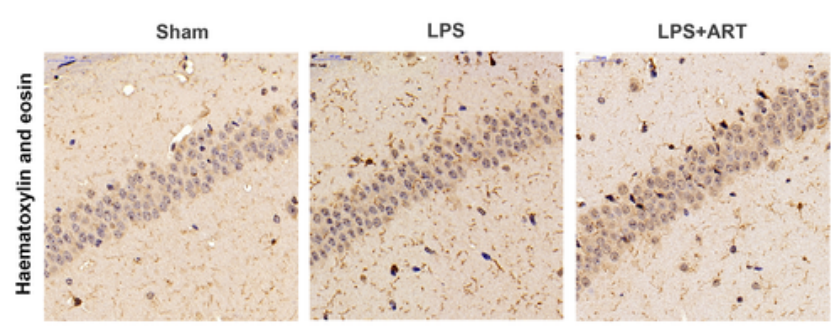

(F)
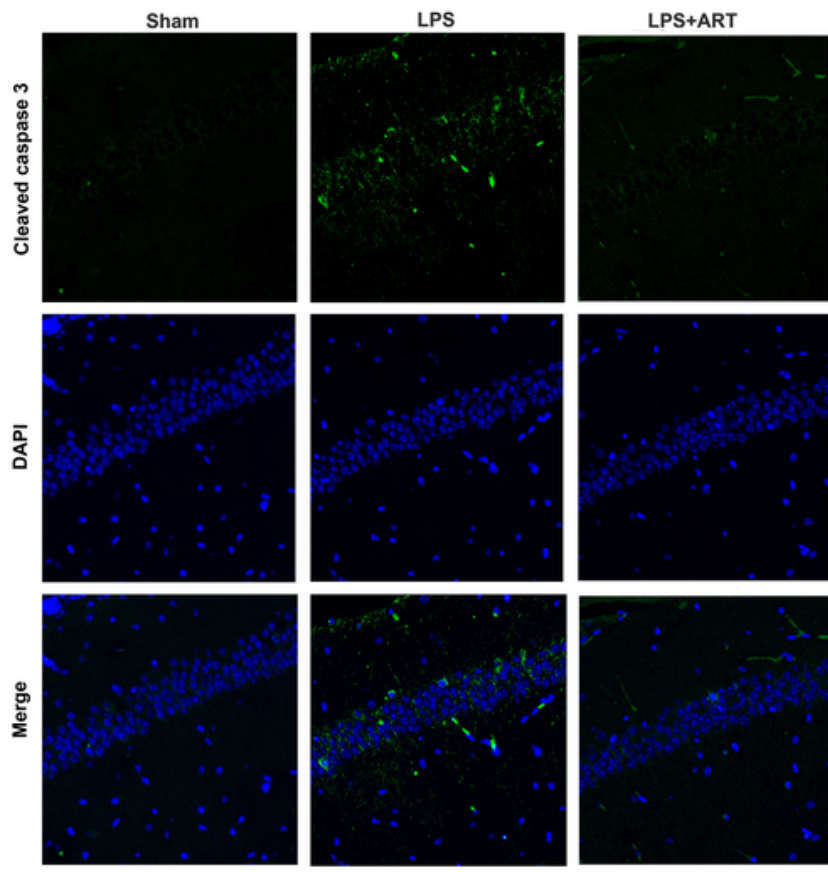

(B)
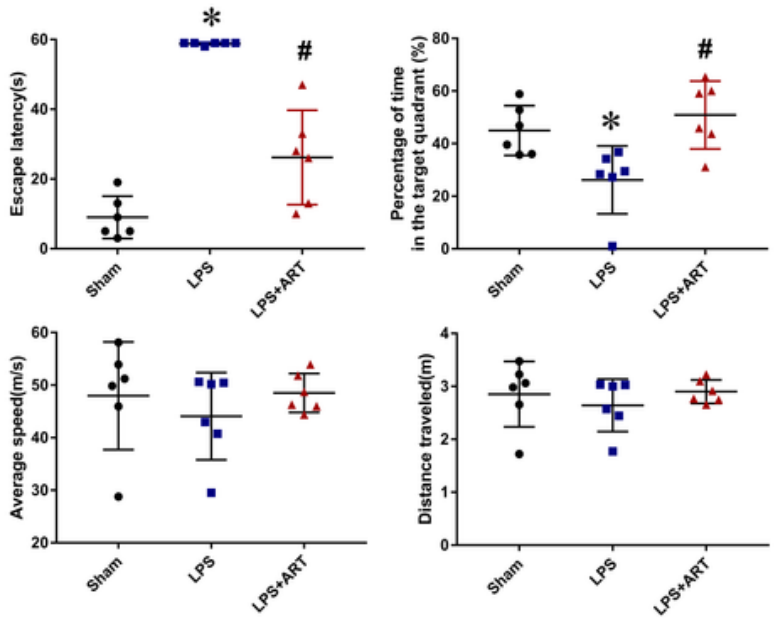

(D)

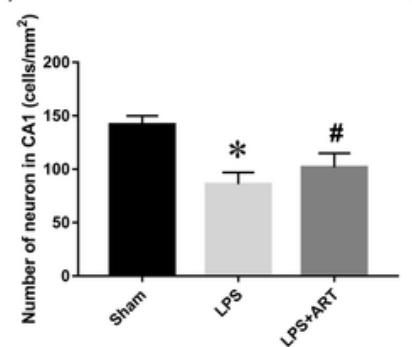

(E)

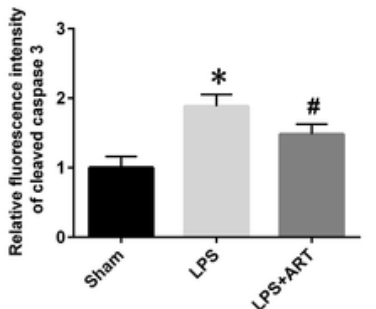

(G)

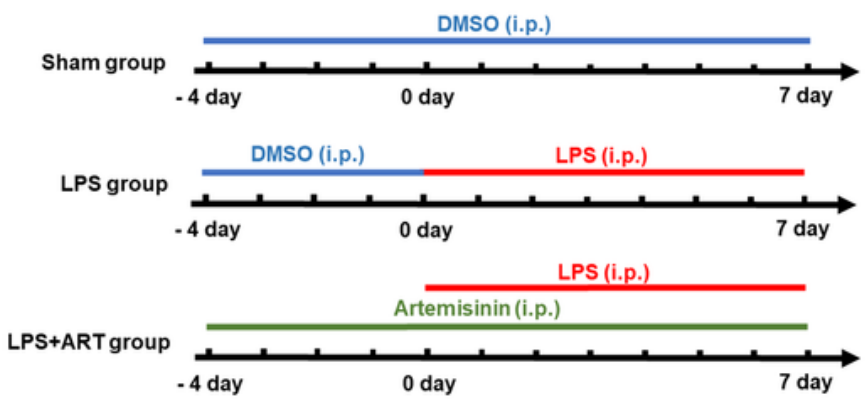

\section{Figure 1}

Artemisinin ameliorated neuronal cell death and improved cognitive impairment in LPS-induced murine neuroinflammatory model. (A) Representative swim paths obtained in the Morris water maze test. (B) Analysis of data obtained in the Morris water maze test. (C) Immunohistochemical staining was used to detect neuronal survival in the CA1 area of the hippocampus. Magnification: 200x. (D) Quantitative analysis of data from C. (E) Statistical analysis of relative fluorescence intensity of cleaved caspase 3. 
(F) Representative images of cleaved caspase 3 staining. Magnification: 200x. (G) Timeline of the in vivo experimental treatments. ${ }^{*} p<0.05$ versus Sham group mice. \# $p<0.05$ versus LPS-treated mice.
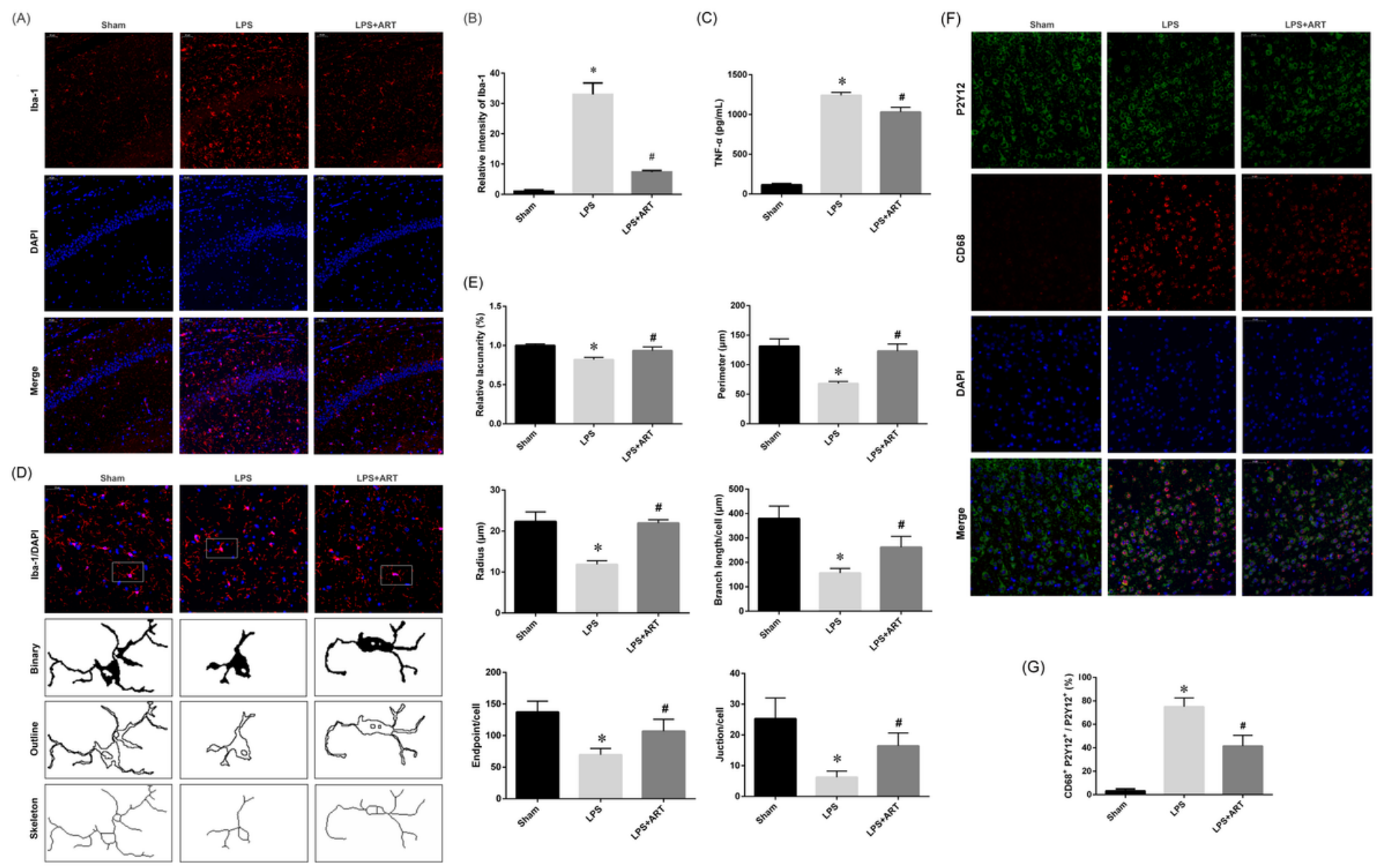

(G)

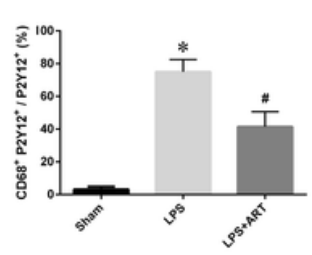

\section{Figure 2}

Artemisinin attenuated LPS-induced microglial activation in the hippocampus. (A) Immunofluorescence staining of Iba-1 positive cells. Magnification: 200x. (B) Quantitative analysis of data from A. (C) The expression levels of TNF- $a$ in hippocampus homogenates were detected by ELISA. (D) Iba-1 staining, binary, outline and skeleton images of the microglia. (E) Statistical analysis of microglia lacunarity, perimeter, radius, branches, endpoint and junctions. (F) Double-staining immunofluorescence of P2Y12 and CD68 in the hippocampus. Magnification: 400x. (G) Quantification of CD68+ and P2Y12+ doublestained cells in the hippocampal CA1. ${ }^{*} p<0.05$ versus Sham group mice. $\# p<0.05$ versus LPS-treated mice. 
(A)

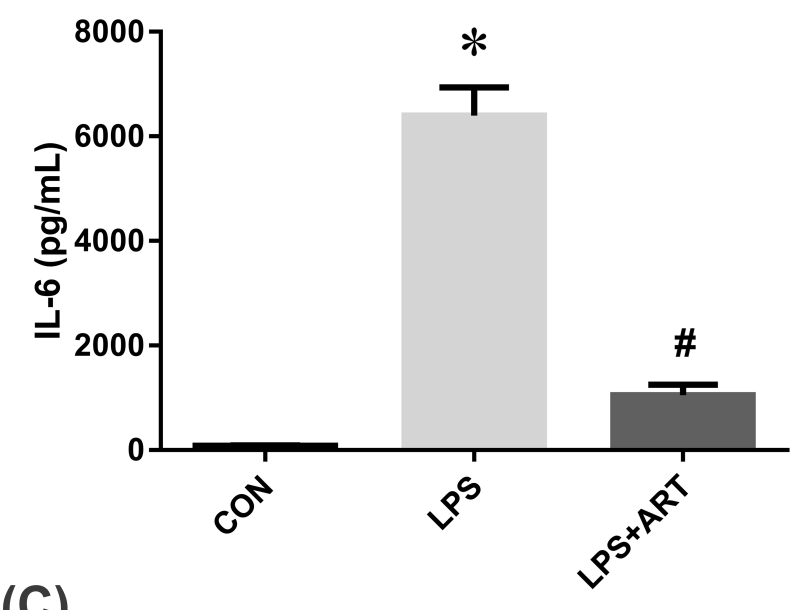

(C)

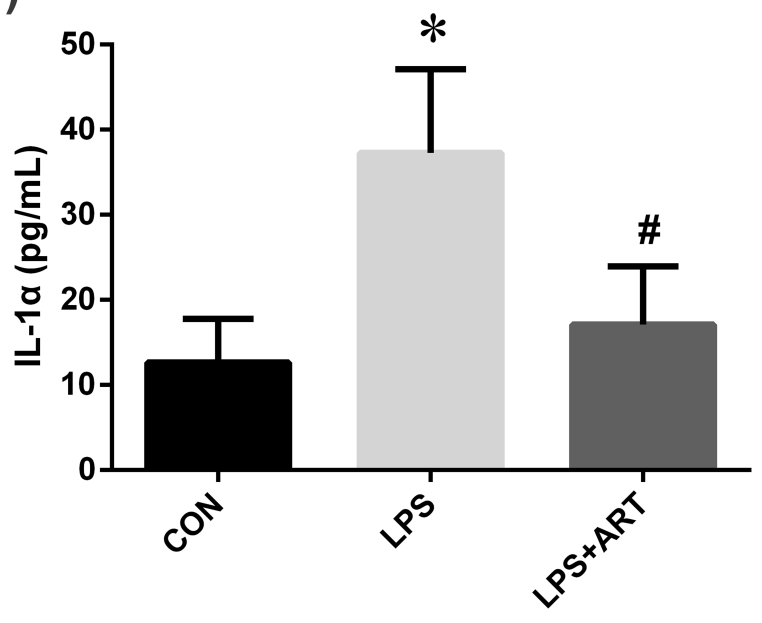

(B)

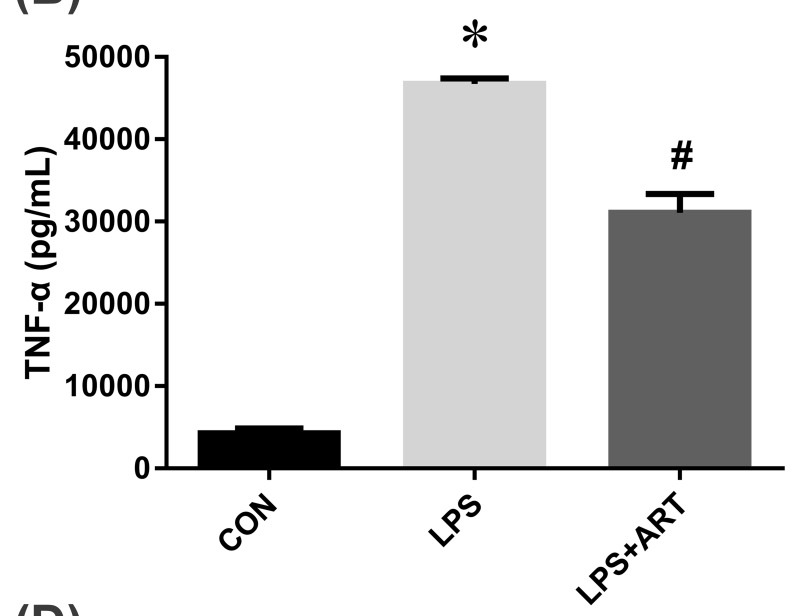

(D)

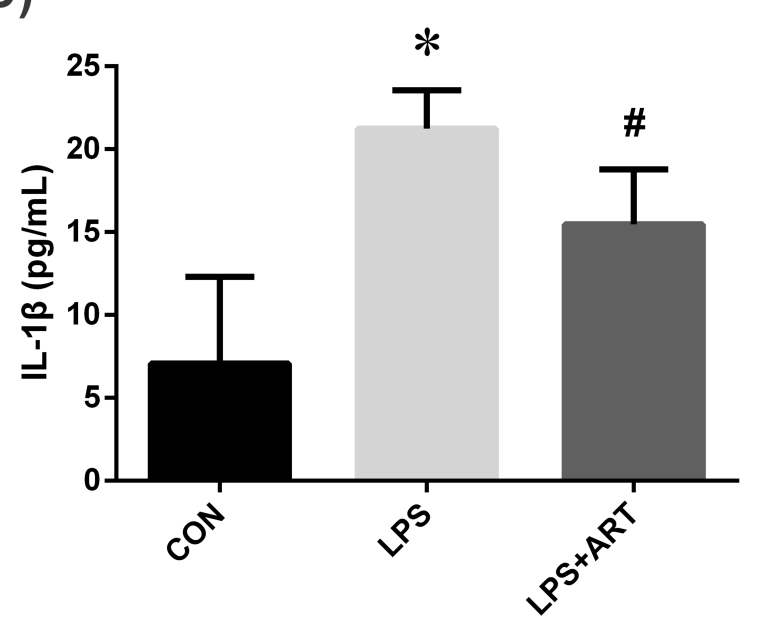

Figure 3

The effects of artemisinin on pro-inflammatory cytokines of BV2 microglia. The levels of the proinflammatory factors IL-6 (A), TNF- $-\mathrm{B}(\mathrm{B}), \mathrm{IL}-1 \mathrm{a}(\mathrm{C})$ and IL-1 $\beta$ (d) were detected by ELISA. * $p<0.05$ versus control group. \# $p<0.05$ versus LPS-treated group. 
(A)

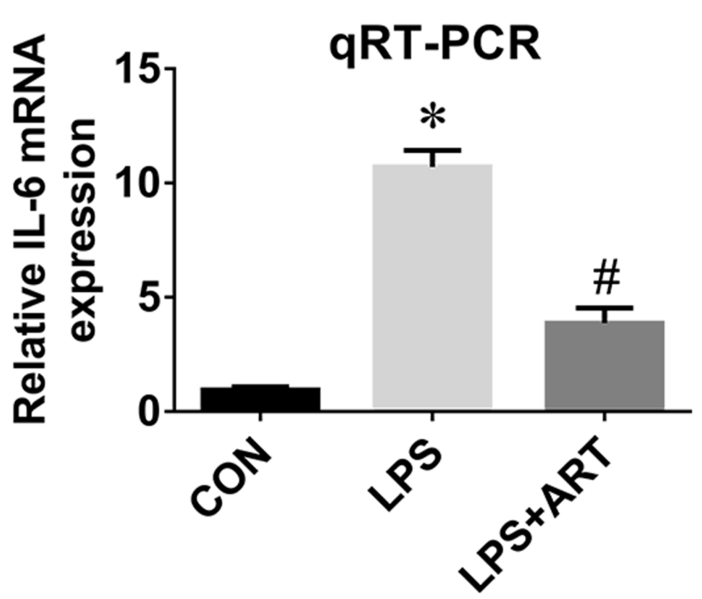

(C)

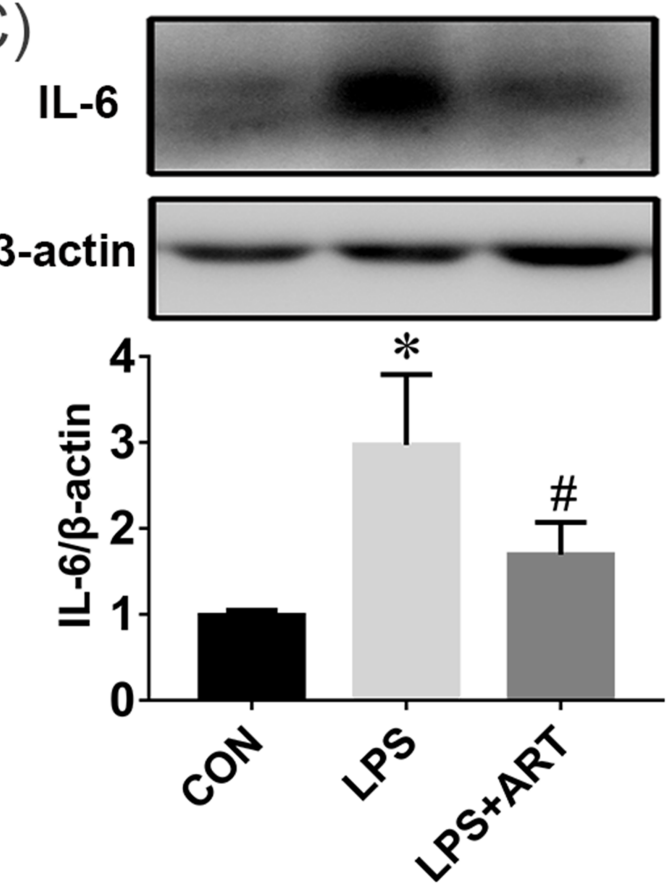

(B)

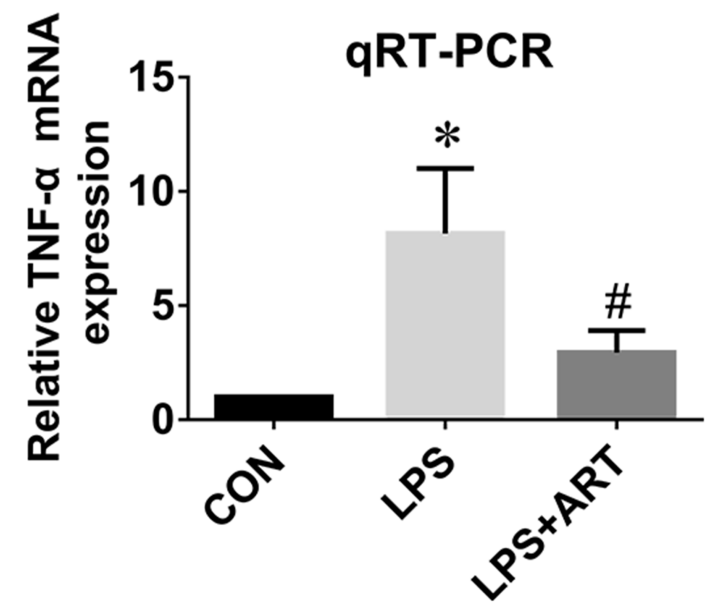

(D)

$24 \mathrm{kDa}$

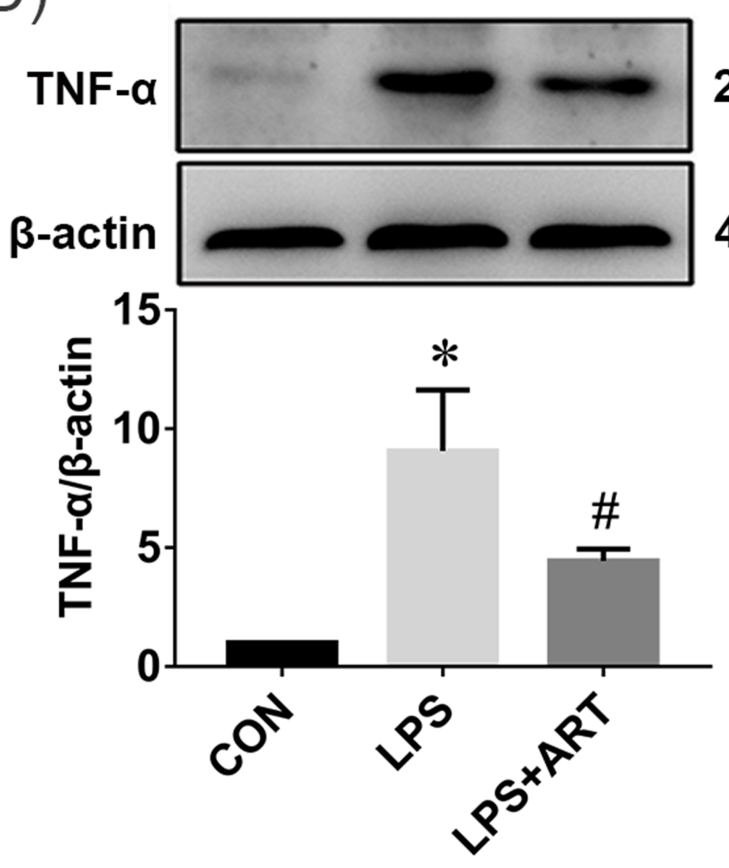

Figure 4

Artemisinin preconditioning reduced LPS-induced upregulation of mRNA and protein expression of IL-6 and TNF-a. (A) The fold changes of IL- 6 estimated by qRT-PCR. (B) The fold changes of TNF-a estimated by qRT-PCR. (C) The level of IL-6 protein detected by western blotting. (D) The levels of TNF-a protein detected by western blotting. ${ }^{*} p<0.05$ versus control group. $\# p<0.05$ versus LPS-treated group. 
(A)
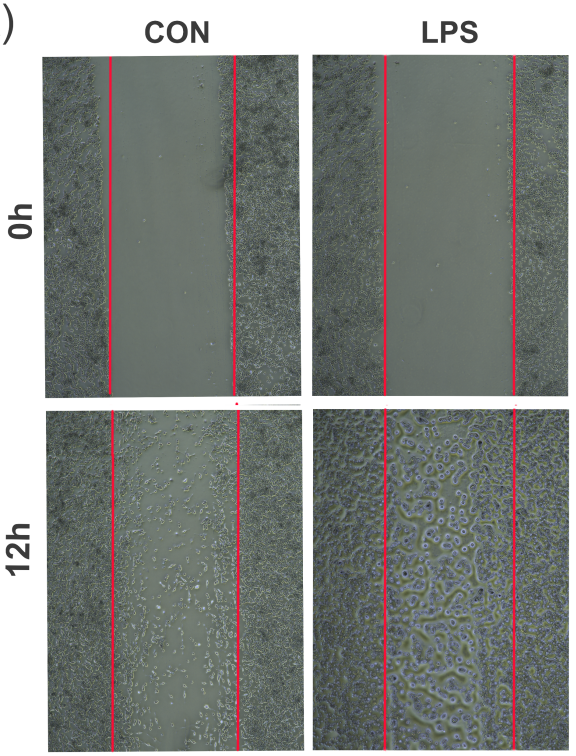

(C)

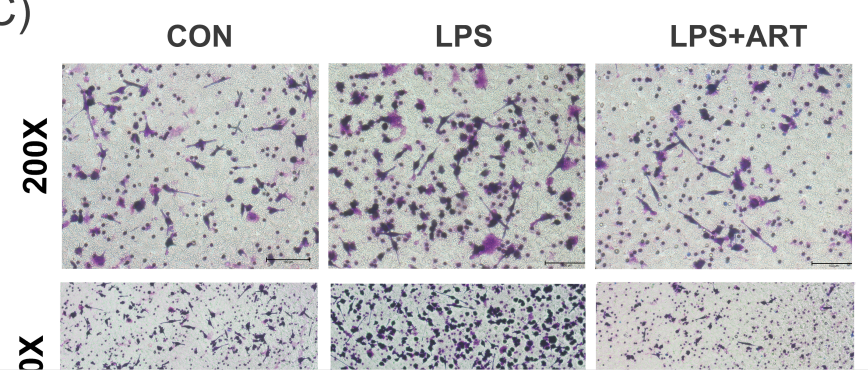

(B)

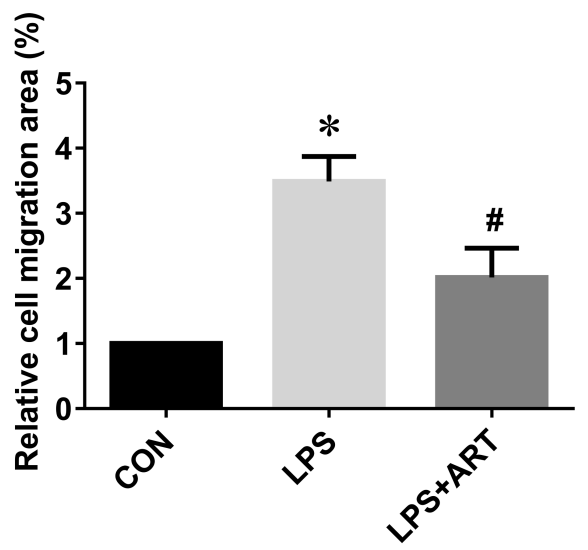

(D)

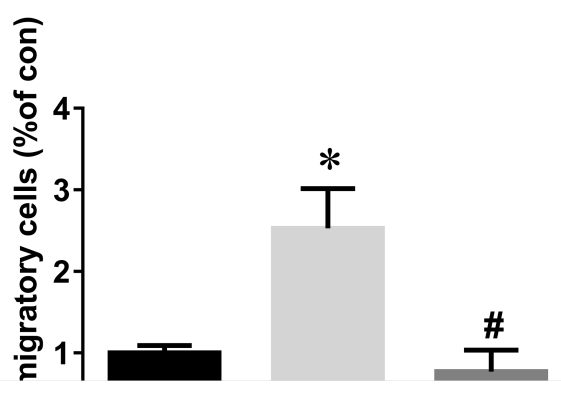

\section{Figure 5}

Artemisinin inhibited the migratory ability of BV2 microglia. (A) Wound healing assay. (B) Relative cell migration area 12 hours after wounding. (C) Transwell chamber migration of BV2 microglial cells. Magnification: 100 or 200x. (D) The relative migratory cells in the lower chamber. (E) The levels of the chemokines MCP-1 were detected by ELISA. (F) The levels of the chemokines MIP-2 were detected by ELISA. * $p<0.05$ versus control group. \# $p<0.05$ versus LPS-treated group. 
(A)

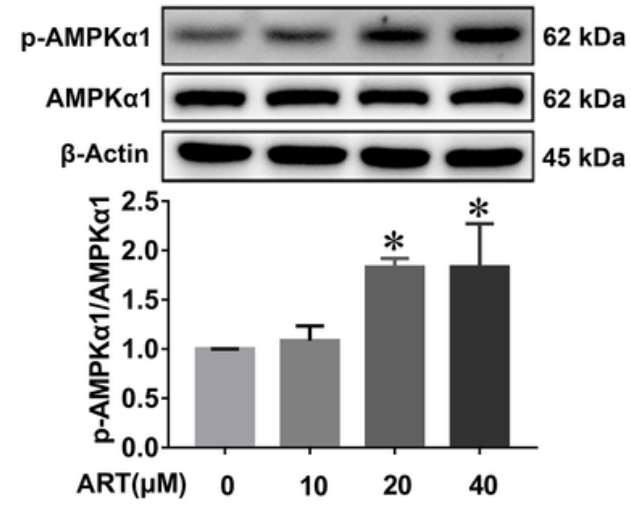

(C)
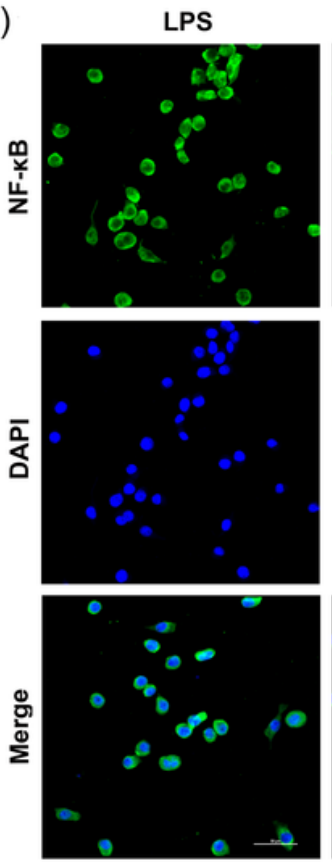

(B)
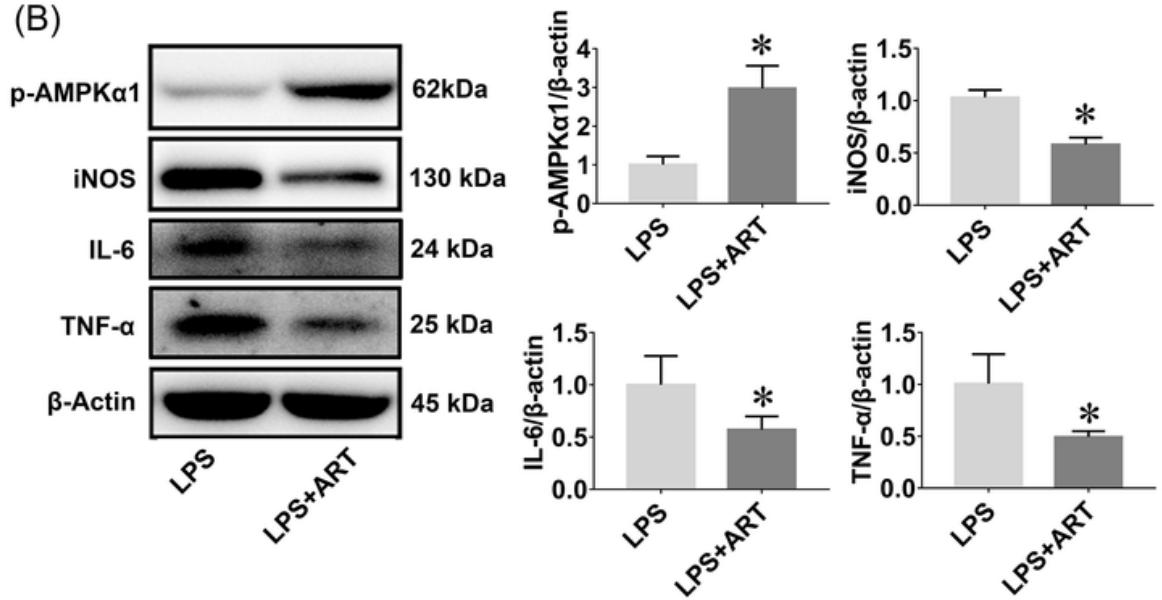

(D)
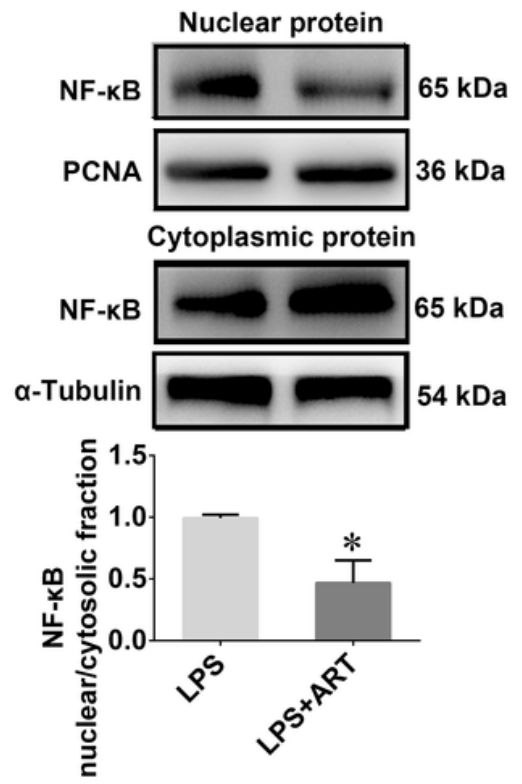

Figure 6

Artemisinin activates AMPKa1 and suppresses the inflammatory response in BV2 cells. (A) Western blots showed that treatment with artemisinin increased the phosphorylation of AMPKa1. (B) Protein levels of AMPKa1, iNOS, IL-6 and TNF-a were detected by western blotting. (C) NF-KB cellular localization analysis by immunocytochemical staining. Scale bars $=50 \mu \mathrm{m}$. (D) Nuclear and cytoplasmic fractions of NF-KB were analyzed through a western blot assay. ${ }^{*} p<0.05$ versus control group. $\# p<0.05$ versus LPS-treated group. 
(A)

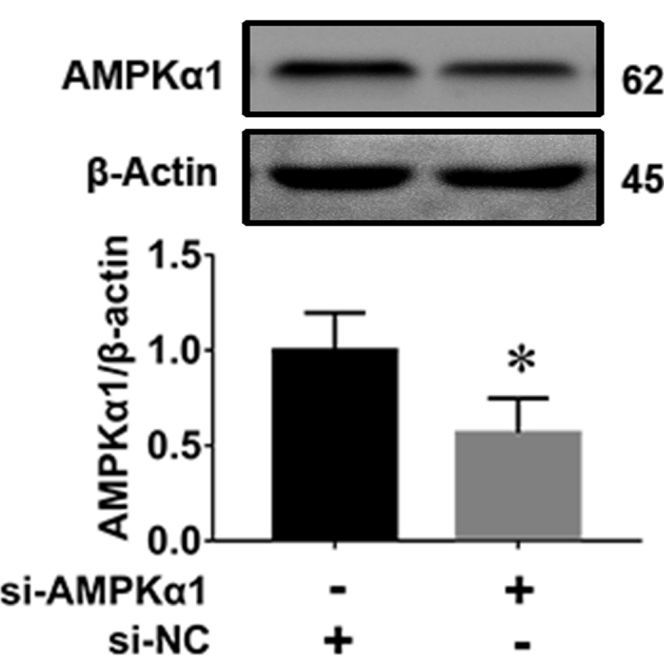

(C)

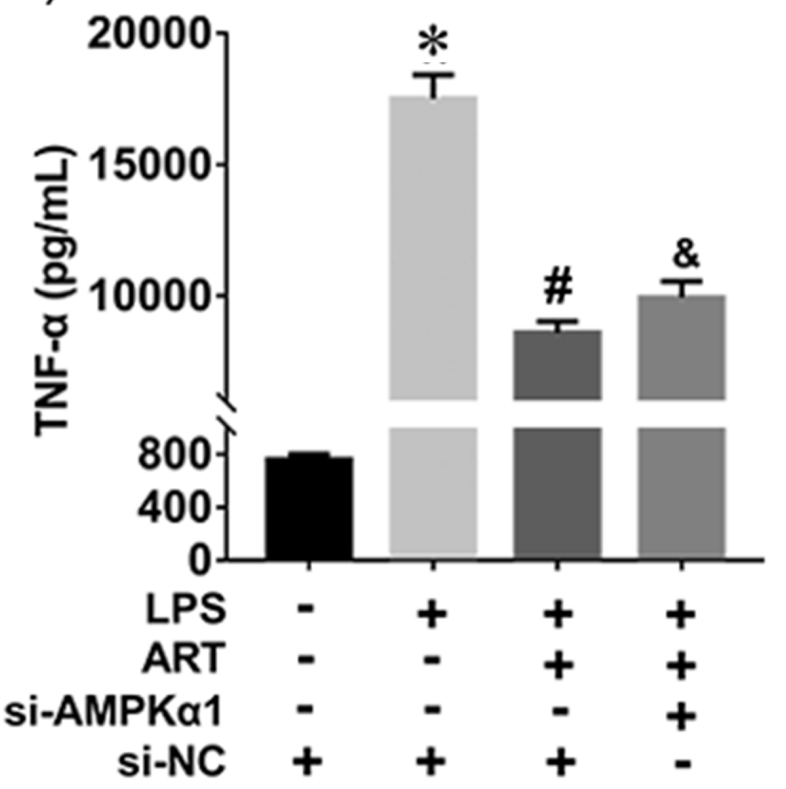

(B)
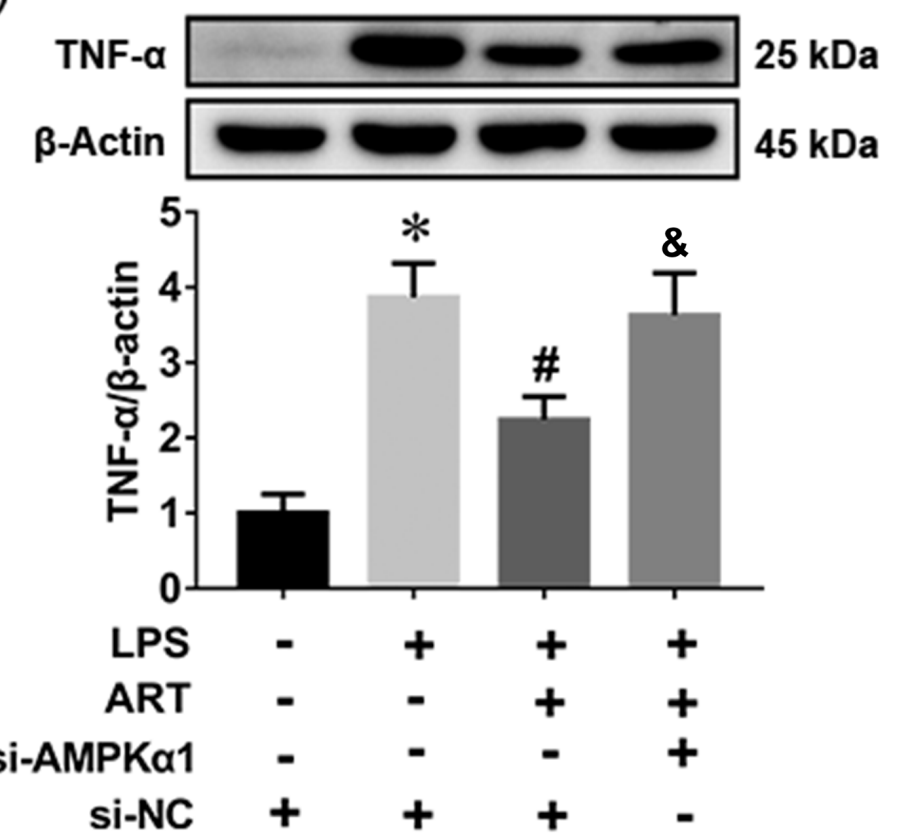

(D)

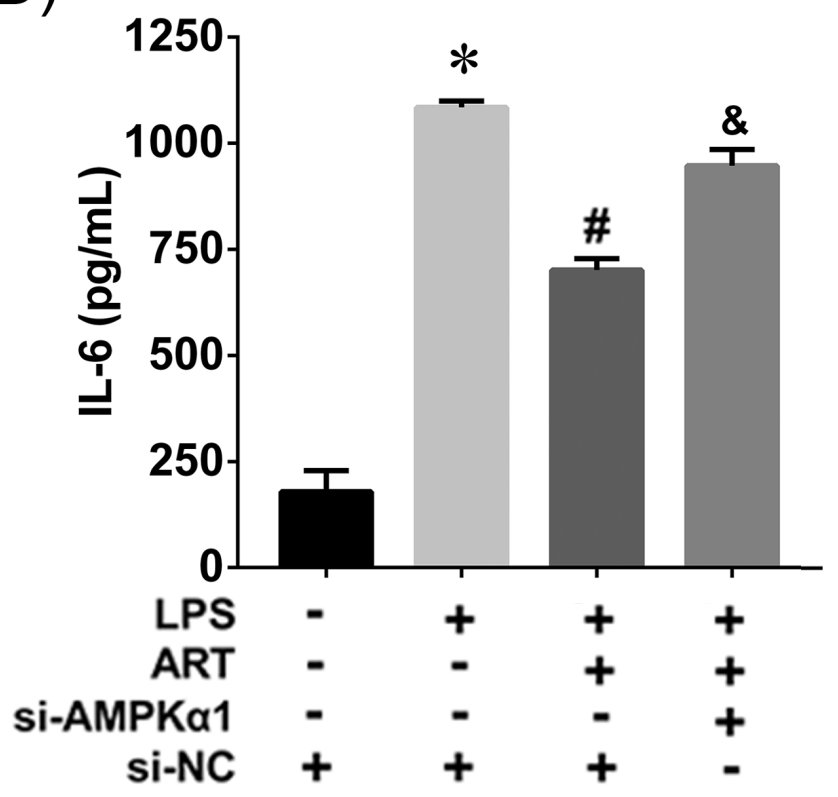

Figure 7

The anti-inflammatory effect of artemisinin in LPS-stimulated BV2-microglia was blocked by siRNA specific to AMPKa1. (A) Efficiency of AMPKa1 knock-down by using siRNA in BV2 cells. ${ }^{*} P<0.05$ versus siNC group. (B) TNF-a protein levels after siRNA treatment in BV2 cells. (C) TNF-a production after siRNA treatment in BV2 cells. (D) IL-6 production after siRNA treatment in BV2 cells. * $p<0.05$ versus control group. \# $p<0.05$ versus LPS-treated group. \& $p<0.05$ versus LPS+ART group. 


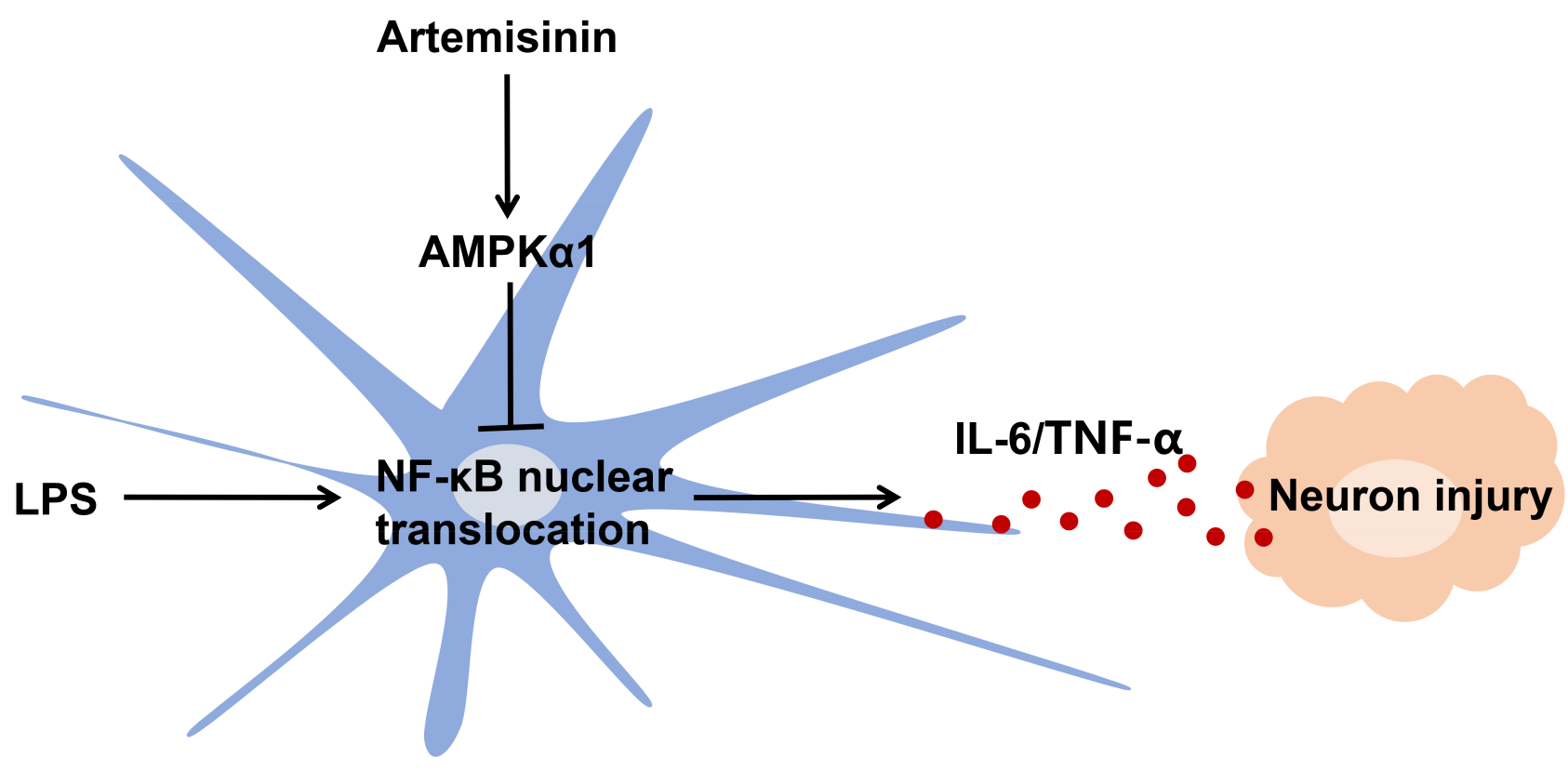

Activated microglia

Figure 8

A model illustrating the mechanism of artemisinin in the treatment of neurocognitive deficits associated with sepsis.

\section{Supplementary Files}

This is a list of supplementary files associated with this preprint. Click to download.

- Fig.S1.tif 